\title{
How Do Dancers Learn To Dance?
}

\author{
A first-person perspective of dance acquisition by expert contemporary dancers \\ Jean-Philippe Rivière Sarah Fdili Alaoui Baptiste Caramiaux Wendy Mackay \\ Univ. Paris-Sud, CNRS, \\ Inria, Université Paris-Saclay \\ F-91400 Orsay, France \\ \{riviere,caramiaux,saralaoui,mackay\}@lri.fr
}

\begin{abstract}
We are interested in supporting motor skill acquisition in highly creative skilled practices such as dance. We conducted semi-structured interviews with 11 professional dancers to better understand how they learn new dance movements. We found that each dancer engages in a set of operations, including imitation, segmentation, marking, or applying movement variations. We also found a progression in learning dance movements composed of three steps consistently reported by dancers: analysis, integration and personalization. In this study, we aim to provide an empirical foundation to better understand the acquisition of dance movements from the perspective of the learner.
\end{abstract}

\section{ACM Classification Keywords}

H.5.m. Information Interfaces and Presentation (e.g. HCI): Miscellaneous; J.5 Arts And Humanities: Performing arts (e.g., dance, music)

\section{Author Keywords}

dance, motor learning, skill acquisition, first-person perspective

\section{INTRODUCTION}

Dance involves some of the richest movement-related skills that a human can achieve. Dancers have to combine athletic performance, in order to execute complex dance techniques, with expression and aesthetics. Reaching such level of expertise in movement production demands an extensive practice of a wide variety of movements in addition to feedback from educators and peers. Nonetheless, the ways in which dancers perceive their learning pathways and the mechanisms involved remain poorly understood.

Understanding how dancers learn movements can be approached through the lens of motor skill acquisition, a set of processes by which one is able, through practice, to perform motor tasks better, faster, and more accurately than "baseline"

Authors' version. Paper published at MOCO 2018. 5th International Conference on Movement and Computing. June 28-30, 2018, Genoa, Italy.

DOI: https: //doi .org/10.1145/3212721.3212723
[26]. Baseline should be understood as the performance level of the same motor tasks by any individuals that would perform them. Hence, motor skill learning relies intensively on practice, which means the repetition of a motor task and its variations.

According to Ericsson, the acquisition of expert skills such as those of music and dance involves a certain type of practice that he calls "deliberate practice", which is the "individuals' prolonged efforts to improve performance" [9]. Therefore, expert skill learners are not only "doing" motor tasks but they are deliberately and intentionally engaged in performing them with attention and motivation.

In the context of dance, movement acquisition relies on practice that can be heavily influenced by traditions carried by the dance educator [8] and the type of dance [17]. As Enghauser states "these traditions come to represent the accepted practices, standards, and overall identity of dance learning and teaching". Another difficulty for dance educators is that dancers have different learning styles and different prior knowledge which requires individualized instructions and approaches to practice [30].

The literature on dance pedagogy is primarily focused either on the perspective of the teacher or on the impact of motor skill acquisition. However, discussion of the practical results of the techniques and mechanisms in play during the training of dancers is largely neglected in the literature.

We propose that the study of dance movement acquisition should begin with an understanding of how dancers perceive their own learning pathways.

To tackle this lack of empirical work we propose to study the perspective of professional contemporary dancers during dance movement acquisition. Our contributions are two-fold: 1) We identify a set of learning techniques used by dancers during their training and 2) we highlight consistent steps and mechanisms involved in the learning pathways of different dancers.

The paper is organized as follows. In the next section we review related work on motor skill learning applied to dance and qualitative methodology to investigate dance acquisition. We then describe our method, which relies on semi-structured interviews and grounded theory analysis. We report the results on the learning processes we obtained through the interviews. 
Finally, we discuss the results and how they can inform the design of learning support technologies.

\section{RELATED WORK}

\section{Motor skill learning in dance}

Nemecek et al. detailed and cataloged peer-reviewed articles related to teaching and techniques in dance medicine and science [20]. They reviewed 59 peer-reviewed publications and organized them into three categories: analytical studies, descriptive studies and experimental studies. From these categories, they drew up three tables. In each table, they classified papers under four main themes: participant health, teaching effectiveness, quality of learning and performance, and the field of dance education. In the category, analytical studies we found publications focused on findings and principles from motor skill learning fields to provide practical knowledge and applications to the dance class. According to Krasnow et al. [19] one of the challenge of bringing motor skill principles to dance class is to "enhance the dance technique class without altering the artistic goal of movements". They explored practical applications for the dance educator. For example, the authors encouraged teachers to explore known movements with dancers. This statement is based on the fact that contextual variety improves both the transfer and retention of learned skills [32]. Also based on motor skill learning findings, [8] focus on improving traditional pedagogical practices. Enghauser argued that new findings in motor skill learning can benefit dance pedagogy. She discussed the following concepts: limited attention capacity, focus of attention, bilateral transfer, practice scheduling, feedback, errors, and learning styles. She aimed at provoking further discussion and research on traditions and practices within the dance class. These two papers, based on motor skill learning principles provide practical tools for dance teachers and dancers but do not provide a concrete view of the experience of dancers and their learning practice.

More focused on dancers, Wilmerding et al. [30] proposed the existence of various stages in the process of learning dance movements: "attention and observation", "replication", "feedback" and "repetition". However, these insights on dance learning were not fully supported by evidence nor by data from educators or learners. In a recent work, Kirsh [18] conducted an experiment where he compared the effect of several factors on movement performance quality: marking, which is performing the movement "in a less than complete manner" ([18], p.3); full-out practice, which is performing the whole movement (replication); and mental simulation of the movement. The study was conducted with dancers from the McGregor company. The author found, within the specific context of this dance company, that marking is the most effective overall strategy to improve technicality, memory and timing. This study compared three learning techniques, but does not provide insight into other techniques that could be used by dancers.

Rather than basing our approach on existing scientific principles, we seek to understand the perspective of the dancers on their learning pathways.
Qualitative methodology to investigate dance acquisition The work of Pierre Vermersch on explicitation technique [29] allows access to, and articulation of, subjective experiences through interviews.

Anne Cazemajou has applied explicitation technique to guide students towards the evocation of their experience of a dance class situation [5]. She takes the example of a dancer, and shows how she guided her step by step through the description of what she had done and experienced during a dance practice. Also based on Vermersch's technique, Françoise et al. collected unique experiences and detailed insights of singular body experiences in dance exploration sessions [13].

Other techniques such as the critical incident technique [11] have been used to determine the effectiveness of feedback in modern dance[12]. In this study, 205 students completed an open questionnaire related to the effectiveness of the latest correction feedback they received from a teacher. From these responses, the author identified 18 strategies of correction perceived as efficient by dancers and 9 as inefficient. Critical incident technique helped dancers to recall and reflect on their experiences.

In this paper, we propose to investigate dance movements acquisition by relying on the learners' (the dancers) singular experiences (first person perspective) through interviews based on critical incident technique. From the collected experiences, our ambition is to better understand the different techniques used by dancers during their learning of dance movements, the mechanisms that occur during their training and the different steps and progressions that form their learning pathway.

\section{METHOD}

We conducted a user-study on dance movement acquisition. We elicited feedback from professional contemporary dancers through semi-structured interviews. With this study, we aim to present the various techniques used by dancers during their training, the mechanisms that appear during the learning process and the learning steps and progression.

\section{Participants}

We recruited 11 professional contemporary dancers (six women; five men) with 7 to 34 years of experience ( $M=18.3$, $\mathrm{SD}=8.3$ ). The participants were recruited through our contacts and during an international dance event in the Centre National de la Danse (CND) that took place in Paris: Camping $2017^{1}$. The participants were not financially compensated. We chose to interview professional dancers due to their extensive experience taking dance classes. During their career they faced, several times, the challenge of learning imposed movements. Moreover, compared to novices, dancers with this expertise have developed an acute body appreciation [28].

\section{Procedure}

We designed and conducted a series of semi-structured interviews focused on the perspective of dancers learning and practicing movement. We used a variation of the Flanagan's Critical Incident Technique [11] introduced by Mackay [21]

\footnotetext{
${ }^{1}$ https://www.cnd.fr/fr/page/298-camping-2017
} 
for HCI, and subsequently used as an input to design [2] and evaluation [15]. Critical Incident Technique facilitates participants recalling situations and describing why they may be atypical. We applied the technique and asked the participants at the beginning of the interview to recall the last time they had to learn a new dance movement in order to retrieve specific examples and avoid generic answers. The interviews were built around 4 topics through 4 main questions:

- Learning Steps: "Can you explain how you learn a dance movement step by step? What is the most important step?"

- Movement Transformation: "Do you make any changes in your movements during the training and why?"

- Understanding of the learning endpoint: "When do you consider the movement to be learned?"

- Using additional information: "Are you using any cues or feedback to learn the movement?"

Each interview was conducted face to face in different venues in France ( 8 in Paris and 3 in Toulouse) and lasted for approximately 30 minutes.

\section{Analysis}

We recorded audio and took handwritten notes during the interviews. We performed a grounded theory analysis [14] from the corpus of the data collected in order to identify, analyse and report concepts within the data from the interviews. The first author transcribed the interviews. Then, two of the authors of the paper read the transcription and highlighted relevant strategies reported by the participants on their learning process of dance movement. This highlighting process produced the 'initial codes' of the data.

We annotated the initial codes on post-its using words from the participants' subjective verbalization. We displayed the post-its (physical annotations) on a large plane surface and grouped those related to each other (see example in Figure 1). Displaying 'post-its' added flexibility, allowed collaboration between the authors, and enabled us to cross-verify our coding, annotations, and clusters. Each cluster formed a concept that we named with terms that summarize them. We finally grouped the concepts into larger categories. These categories are complementary and provide a way of articulating the data collected and the concepts identified. These categories offer a vision of a dancer's learning journey. In the example depicted in figure 1, each column represent a concept, the 'post-its' in top of each row represent the name of each concept (Observation, Imitation, marking and segmentation), post-it below are summaries of dancers' sentences. These 4 concepts represent the category Techniques of Learning.

Following [6], we did not wait until data were completely collected to begin the data analysis. We followed three iterations: the first iteration was performed on responses from the first 4 participants, then we iterated on the following 4 , and lastly we performed the analysis with responses from the last 3 participants. These iterations allowed us to refine our analysis twice, to verify it with further participants and to guide subsequent data collection. For example, after the 4th interview, in

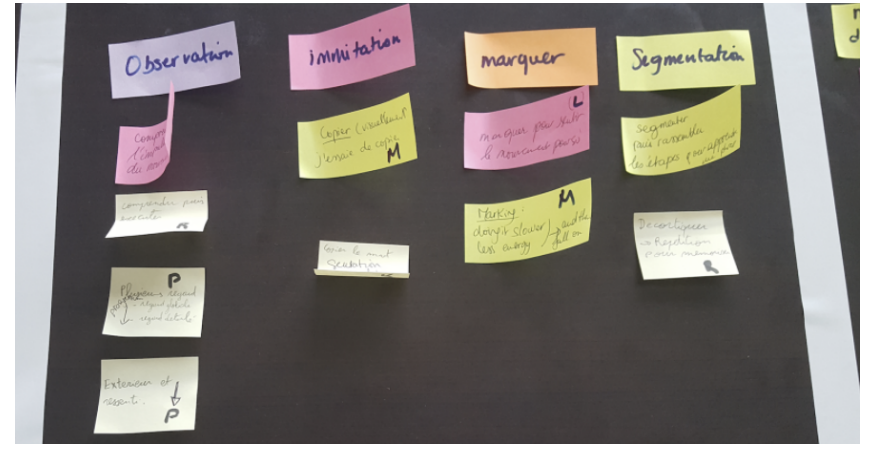

Figure 1. First iteration of the grounded analysis. Initial codes from the interviews are clustered in column. A term on top of each column is generated to describe the concept described by the codes, such as observation, or imitation. A category can then be defined gathering this set of four concepts.

order to retrieve more specific data on variations, we refined the questions "Do you use the variations of your movement to learn more easily?" to "Do you make any changes in your movements during training and why?".

In the following, we anonymised the interviews and refer to the dancers as participant 1 to 11 , denoted by P1 to P11.

\section{RESULTS}

We identified several concepts that we were consistently able to elicit from the dancers. These responses were grouped into two categories. The first category, termed techniques of learning, groups the concepts that are actions that dancers may decide to use. For instance, imitation (the replication of the target movement) is a learning technique. The second category, termed learning progression, brings together the concepts of steps and mechanisms that take place over time. In this section we first describe the concepts within each category: techniques of learning and learning progression.

\section{Techniques of learning}

During the learning process, dancers use various techniques that consist of specific actions that allow them to acquire movements. We identified the following concepts as techniques: $o b$ servation, repetition, imitation, marking, segmentation, mental simulation and personal adaptation.

\section{Observation}

All of the participants reported the observation of the movement as the very first action of the learning process. A dancer can observe the movement according to several levels of detail. The participants mentioned that they observe "the energy" (4/11), "the rhythm" (4/11), "the space" (4/11), "the direction" (P6,P10), "the impulse" (P3), "the form" (P9), "the musicality" (P11), or "the orientation" (P10). Observation is an iterative action and is carried out throughout the learning process:

I'm trying to see the big picture, I'm looking at it from different angles. You are brought to see it [the movement] several times. The first look is a global look, I look at the energy, the situation in space, the most important ones. My second look will focus on the details and see how the 
energy flows, what is the initiation of the motion in the phrase. (P3)

A dancer can also observe various isolated elements that contribute together to the global movement.

Firstly it's observation, I observe the teacher, the dancer or the choreographer. I observe the movement and then I try to understand, how the movement is technically constructed, then I look at the rhythm, the musicality, the energy. (P11)

\section{Repetition}

Dancers constantly mentioned repetition as the way to progress in learning. For P11, the movement becomes automatic thanks to repetition: "we do it several times until it becomes an automatism". The repetition of a movement can last several days; P5 mentioned 4 to 5 days to reach the perfect movement. P7 describes the repetition of the movement as longitudinal to the learning that can "last as long as you want" (P7) and depends on the "level of requirement" (P7).

\section{Imitation}

All participants try to imitate the reference movement identically "The most similar possible, otherwise it's not the same. I'm trying to make the repetition as accurate as possible until it's assimilated." (P1). The criterion of success is based on how similar the movement is to the reference in its global form.

\section{Marking}

More than half $(7 / 11)$ of the participants reported that they decompose the reference movement to work independently on "space" (P5, P6, P7, P9), or "time" (P7, P9). P2 described starting their training with a smaller movement, with less energy or with one part of the body. P8 compared marking to "sketching the movement".

There are also other parameters to adjust, when realizing a choreography, there are others dancers, the space I use, the one we share, the time I have and the one we share. And I want to work on these things in isolations. That's why the movement is therefore marked. (P7)

\section{Segmentation}

More than half (6/11) of the participants reported to decompose the reference movement into smaller sequences. (P8) "I will work on elements in isolation and repeat them, more often the problematic ones". These isolated sequences must be understood here as temporal segments of the reference movement that have a clear beginning and end. Segmentation can be used to isolate problematic sequences, work on them separately and recombine the whole movement: "I'm going to work and repeat elements separately, often those that are problematic." (P7).

\section{Personal adaptation}

Five participants mentioned the use of personal adaptation. We call personal adaptation the explicit variations used to make a movement easier to execute. Personal adaptations appear when the dancer has difficulties to produce a movement. In this case, the dancer can modify the movement to make it easier to perform, for example, P7 mentioned a fall to the ground she was not able to perform in time because of her height. She used her hands even though they were not part of the reference movement.

\section{Mental simulation}

Three participants refer to mental simulation to support movement memorization. Instead of physically executing the movement, dancer can mentally simulate the movement: "sometimes, during the evenings, in bed, I like to go over the whole piece but, in my head. I see myself dancing" (P5).

Figure 2 depicts these techniques with regards to the reference movement to be learned.

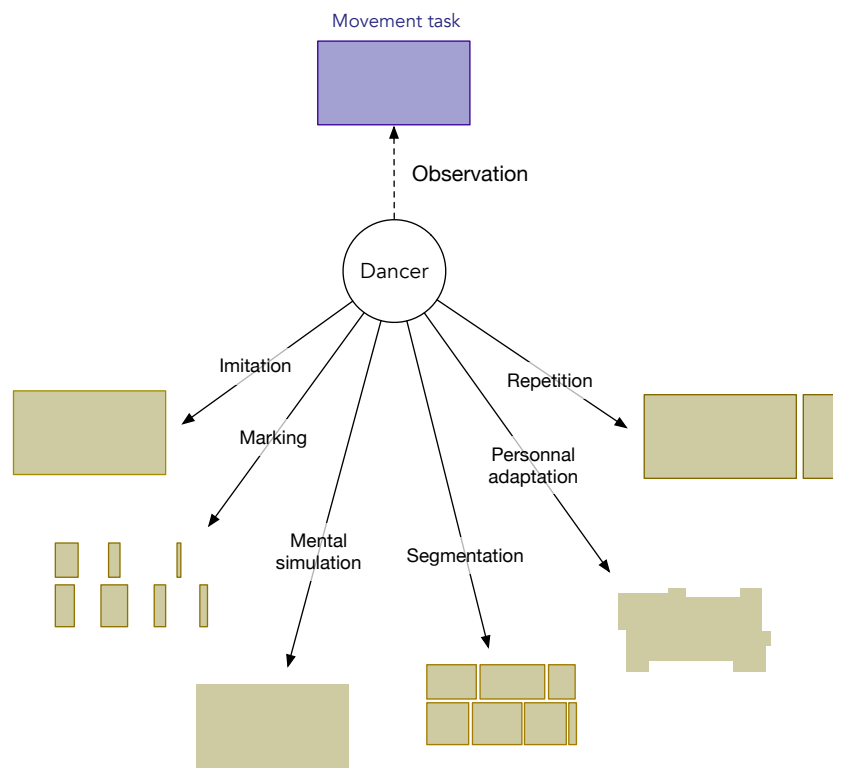

Figure 2. The different techniques used by the dancers during dance movement acquisition. In the task of learning a new movement (purple square on top), the dancer uses movement observation, imitation, marking, mental simulation, segmentation and variations (see descriptions in text).

\section{Learning progression}

Our second category reveals the progression in learning dance movements. We identified the following concepts: analysis, integration, fluidity, personalization and implicit variations.

\section{Analysis}

All the participants reported that the first step in the learning of dance movement is an analysis step that includes several actions ranging from observing the movement to replicating it. Importantly, the actions of the dancers seem to be made with the goal of reducing the complexity of the movement at the beginning and gradually increasing it:

In the beginning, it's just the frame. As I move forward, elements will be added. I'll do it chronologically. I refine every movement. (P6)

Therefore, complexity seems to be understood as the level of detail in the movement, which is increased from a coarse 
analysis, "the frame" (P6), to further refinements through the addition of details.

\section{Integration}

Almost all participants (10/11) reported that, through repetition, the movement gradually becomes integrated into the body. The word "integration" was used to refer to an incorporation of movement at the cognitive level but also at the motor level. A movement is perceived as being learned when the cognitive load, exerted by the dancer during the movement execution, is reduced:

[I know that I integrated a movement] when I can reproduce it naturally. Naturally, because the movement is inside of my body. I don't need to think about it anymore. It's more the body that goes and realizes the movement. (P3)

\section{Fluidity}

Movement integration was reported by the dancers through the resulting movement qualities: "natural" (P7, P3), "fluid" (P3) or "organic" (P2). Most of the dancers (6/11) reported that movement has been integrated when it is smooth, fluid and they don't have to think about it:

You know a movement when it is integrated into your body and you don't need to think anymore. When you don't think about it, when it comes out smoothly, just by pressing the start button. (P7)

\section{Personalization}

Seven participants reported personalizing the movement. This relies on changes that the dancers apply to appropriate the movement according to their individualities such as body characteristics or expressive range. Personalization is the moment when dancer can "deconstruct, deform and give qualities" (P11), "bring his own touch" (P1) or "doing it according to your body your ability, your sensation and your feeling" (P9). According to $\mathrm{P} 4$, this step has an active role in learning: "There is no learning without appropriation". One of the characteristic of personalization is the use of specific explicit variations. Seven participants reported adding explicit variations only when the movement is integrated. We call expressive variations, these explicit variations used by a dancer as a means of expression. P2 stated that expressive variations are a way to go beyond what they have learned in dance classes, to "step outside the framework of dance class" and "take liberties". These variations bring "different intensities, subtleties, movement qualities or interpretations" (P2).

\section{Implicit variations}

Almost all participants (8/11) reported that implicit variations appear all along the process of dance movements acquisition. These variations perceived by participants are non-volitional actions, in other word, variations that are not controlled by the dancers. P8 perceived them as personal body signature "We all have some kind of body signature that is different from others" (P8). These signatures characterize the dancer and are present across the different steps of learning "To some extent, there are always changes brought to the movements. Movements are transformed because different bodies produce it." (P7). P2 argues that it takes years of practice to shape a body and that this shaping has an effect on the movement: "It takes years of repetition, the body is forming and there's body memory.". Finally, dancers also perceived that the movement has an inherent trial-to-trial variability.

The movement moves all the time even when you feel like you're learning the same thing, it's never fixed... The same movement won't be the same thing. Because you matter, your body. As a dancer you evolve. (P11)

\section{DISCUSSION}

In this paper, we reported on how expert contemporary dancers describe the way they learn new dance movements, and precisely the learning techniques in play (such as observing, imitating, marking etc) and the way they progress throughout their learning journey.

Learning techniques highlighted by dancers are diverse: from movement observation to explicit variations of the same movement. Altogether, these techniques can be seen as a set of "tools" that dancers can use in practice in order to learn a new dance movement. Interestingly, these techniques operate as variations on the initial movement: marking allows for representing the movement into more compact keyframed actions; segmentation allows for representing parts (or segments) within the movement; while explicit variations allow for changes in amplitude, dynamics or qualities. Previous work in motor skill learning has shown the benefit of variation on retention and transfer [22, 24, 25], which has also been examined in expressive musical performance [4], but never in dance. Also it is worth noting that most of these previous studies have primarily focused on spatio-temporal variations of movement and were not taking into account more complex movement variations such as variations in dynamics or in segments performed. We believe that these results shed light on interesting research directions on the beneficial effect of heterogeneous movement variations on motor learning.

Another result is the elicitation of the learning progression through different phases. Phases in skill development have already been identified in previous work, especially through the well-established behavioral model proposed by Fitts and Posner [10]. Their model includes three main stages in motor skill development: a cognitive stage, an associative stage and an autonomous stage. Our findings on dancers' learning steps are consistent, yet extend, this model. The analysis step identified from dancers' interviews seems to encompass the cognitive and the associative stages: dancers analyze which movement to perform and how to execute it. The integration of movement corresponds to the autonomous phase proposed in the model: a phase characterized by a fluid, reliable, efficient movement and a reduced cognitive load. However, the personalization step does not directly fit Fitts and Posner's model. We believe that movement personalization is a critical step, as reported by the dancers, although its role in motor skill acquisition remains largely unexplored.

Among the highlighted phases, integration is the moment where the dancer "does not need to think about it anymore" and can be viewed through the lens of the notion of embodiment. In the theory of embodied cognition, objects manipulated in 
goal-oriented movements become, through practice, absorbed into the body schema, (i.e. they become an extension of the body [23]). In dance, the movement is reflexive, meaning that the goal of the movement is the movement itself [27]. We can thus relate the moment of integration with the phenomenon of embodiment: it corresponds to the "switch" during which the movement is absorbed and the cognitive load required for movement execution is reduced. From our results, this movement integration is perceived by dancers a posteriori, that is to say once the movement is integrated. They perceive how movement execution is facilitated and requires less cognitive load.

Finally, from what we have seen in our study of a particular community of dancers, the learning practice seems to be composed of multiple iterative processes. Dancers iterate between the learning techniques. They also iterate between steps within the learning progression. For instance, in the analysis phase, there is a back and forth between observation and repetition. Iteratively, dancers build movements by removing or adding segments and details. There seems to be also iterations between steps. For example, a dancer may want to deconstruct or refine an integrated movement. To do so, they may go back to an analysis step. This suggests that a successful practice in dance relies on a complex schedule of techniques and iterations. This leads us to believe that further investigation into whether the learning practices of dancers are necessarily composed of multiple iteration.

In motor skill acquisition, it is sometimes taken for granted that practice is based on full movement performance. Indeed a common experimental paradigm for the study of motor learning is based on motor task repetition and variation of certain factors of practice [26,31]. While this is relevant for a certain class of motor tasks, in dance, our results suggest that a richer repertoire of practice techniques exist. Such repertoire has been built by dancers in order to achieve the performance of the "dance a movement". In related work, Kirsh has already shown that professional dancers are using marking as a way to rehearse previously learned movements [18]. In this paper, we shed light on a broader set of techniques whose impact on learning may be significant and would require a more thorough investigation.

\section{TOWARDS LEARNING-SUPPORT TECHNOLOGIES}

The methodology proposed in this paper is based on firstperson perspective and investigation of experience. We have shown that such methodology, when applied to dance movement learning, is a powerful tool to elicit learning techniques and progression among expert dancers. But the methodology used in this paper has also been shown to be a insightful tool in interaction design (see for instance [16]). Indeed, if we consider the interviewee (the dancer) as a potential user of an interactive system, such methodology allows us to place the experience of the user at the center of the design process of such system.

More precisely, driving the dancer towards a specific experience (of movement learning) helped highlight difficulties and solutions found by dancers at that moment. To do this, we used a technique based on the critical incident technique. Applied in this context, critical incident technique helps identify breakdowns and solutions leading to opportunities for design [21]. In this section we propose to present elements of the results as insights for the design of dance movement learning-support technology.

One important aspect stemming from our results is the variety of techniques used by dancers to acquire a new movement. As we mentioned previously, while dancers seem to use all, or a good part of, these techniques, the way they use them and their ordering seem to remain unique to each dancer. We see this use of learning techniques as a way to drive the design of a learning-support tool towards a modular, toolkit-based software. Elements of the toolkit that would accompany and facilitate certain techniques could be used in an order specified by the user. In our previous work, we have shown that such a toolkit can be easily used by designers and novice practitioners to build movement-based sonic interactions [2].

The idea of a toolkit where dancers can use modules the way they would like sheds light on an important feature of such technology: their capacity to be appropriated. A system should not only support movement analysis features but also movement personalization by allowing the dancers to "step outside the framework", as described by one of the dancer. As Dix stated, "we might not be able to design for the unexpected but we can design to allow the unexpected" [7]. We believe that this could be afforded by allowing variations in the input movements through adaptive interactive systems that are deployable in real-world situations such as dance studios. While input movement variations are usually discarded in interactive systems in order to improve between-user generalizability.

recent approaches propose to use such explicit variations as interaction technique $[3,1]$. This is motivated by the need to augment the expressive bandwidth of movement-based interaction techniques. We embrace these approaches and argue for considering explicit and implicit variations in the design of systems supporting motor skill acquisition.

\section{CONCLUSION}

This paper contributes to a better understanding on how professional contemporary dancers train and master dance movements. Our aim is to use our findings, grounded in dancers' practice, to fuel further research in dance skill learning. First we identified a set of techniques that participants consistently reported during learning. Second, we presented a progression in the learning of dance movement composed of three steps: analysis, integration and personalization. We discussed our two main findings independently and show how they are complementary. Our work suggests that movement dance skill acquisition relies on practice involving heterogeneous variations of the same movement. This result provides an empirical foundation to further research in dance science but also provides insights for the design of learning-support interactive technologies.

\section{ACKNOWLEDGMENTS}

This project has received funding from the European Union's Horizon 2020 research and innovation programme under the Marie Sklodowska-Curie grant agreement No 659232, from 
European Research Council (ERC) grant $\mathrm{n}^{\circ} 321135$ CREATIV: Creating CoAdaptive Human-Computer Partnerships. We thank Marcelo Wanderley and all the study participants for their involvement.

\section{REFERENCES}

1. Jessalyn Alvina, Joseph Malloch, and Wendy E. Mackay. 2016. Expressive Keyboards: Enriching Gesture-Typing on Mobile Devices. In Proceedings of the 29th Annual Symposium on User Interface Software and Technology (UIST '16). ACM, New York, NY, USA, 583-593. DOI : http://dx. doi . org/10.1145/2984511.2984560

2. Baptiste Caramiaux, Alessandro Altavilla, Scott G. Pobiner, and Atau Tanaka. 2015a. Form Follows Sound: Designing Interactions from Sonic Memories. In Proceedings of the 33rd Annual ACM Conference on Human Factors in Computing Systems (CHI '15). ACM, New York, NY, USA, 3943-3952. DOI : http://dx. doi.org/10.1145/2702123.2702515

3. Baptiste Caramiaux, Nicola Montecchio, Atau Tanaka, and Frédéric Bevilacqua. 2015b. Adaptive gesture recognition with variation estimation for interactive systems. ACM Transactions on Interactive Intelligent Systems (TiiS) 4, 4 (2015), 18.

4. Christine E Carter and Jessica A Grahn. 2016. Optimizing Music Learning: Exploring How Blocked and Interleaved Practice Schedules Affect Advanced Performance. Frontiers in Psychology 7 (2016).

5. Anne Cazemajou. 2014. L'explicitation comme pédagogie phénoménologique et support de créativité en danse. (01 2014).

6. Ji Young Cho and Eun-Hee Lee. 2014. Reducing confusion about grounded theory and qualitative content analysis: Similarities and differences. The Qualitative Report 19, 32 (2014), 1.

7. Alan Dix. 2007. Designing for appropriation. In Proceedings of the 21st British HCI Group Annual Conference on People and Computers: HCI... but not as we know it-Volume 2. BCS Learning \& Development Ltd., 27-30.

8. Rebecca Enghauser. 2003. Motor Learning and the Dance Technique Class: Science, Tradition, and Pedagogy. Journal of Dance Education 3, 3 (2003), 87-95. DOI : http://dx. doi . org/10. 1080/15290824.2003.10387235

9. K Anders Ericsson, Ralf T Krampe, and Clemens Tesch-Römer. 1993. The role of deliberate practice in the acquisition of expert performance. Psychological review 100, 3 (1993), 363. DOI :

http://dx.doi.org/10.1037//0033-295X.100.3.363

10. Paul Morris Fitts and Michael I Posner. 1967. Human performance. (1967).

11. John C Flanagan. 1954. The critical incident technique. Psychological bulletin 51, 4 (1954), 327.
12. Sylvie Fortin. 1988. La technique des incidents critiques pour cerner l'efficacité des feed-back de correction en danse moderne. 14 (01 1988).

13. Jules Françoise, Yves Candau, Sarah Fdili Alaoui, and Thecla Schiphorst. 2017. Designing for Kinesthetic Awareness: Revealing User Experiences Through Second-Person Inquiry. In Proceedings of the 2017 CHI Conference on Human Factors in Computing Systems (CHI '17). ACM, New York, NY, USA, 5171-5183. DOI : http://dx . doi . org/10.1145/3025453.3025714

14. Barney Glaser. 2017. Discovery of grounded theory: Strategies for qualitative research. Routledge.

15. H. Rex Hartson and José C. Castillo. 1998. Remote Evaluation for Post-deployment Usability Improvement. In Proceedings of the Working Conference on Advanced Visual Interfaces (AVI '98). ACM, New York, NY, USA, 22-29. DOI: http://dx. doi . org/10.1145/948496.948499

16. Kristina Höök, Baptiste Caramiaux, Cumhur Erkut, Jodi Forlizzi, Nassrin Hajinejad, Michael Haller, Caroline Hummels, Katherine Isbister, Martin Jonsson, George Khut, and others. 2018. Embracing First-Person Perspectives in Soma-Based Design. In Informatics, Vol. 5. Multidisciplinary Digital Publishing Institute, 8 .

17. Janet Karin. 2016. Recontextualizing Dance Skills: Overcoming Impediments to Motor Learning and Expressivity in Ballet Dancers. Frontiers in Psychology 7 (2016), 431. DOI:

http://dx.doi .org/10.3389/fpsyg. 2016.00431

18. David Kirsh. 2013. Embodied Cognition and the Magical Future of Interaction Design. ACM Trans. Comput.-Hum. Interact. 20, 1, Article 3 (April 2013), 30 pages. DOI : http: //dx. doi . org/10.1145/2442106.2442109

19. D.H. Krasnow and Steven Chatfield. 1996. Dance science and the dance technique class. 4 (01 1996), 162-172.

20. Sarah M. Nemecek and Steven Chatfield. 2007. Teaching and Technique in Dance Medicine and Science: A Descriptive Study with Implications for Dance Educators. 7 (10 2007).

21. Wendy E Mackay. 2002. Using video to support interaction design. DVD Tutorial, CHI 2, 5 (2002).

22. Richard A Magill and Kellie G Hall. 1990. A review of the contextual interference effect in motor skill acquisition. Human movement science 9, 3 (1990), 241-289.

23. Alva Noë. 2004. Action in perception. MIT press.

24. Richard A Schmidt. 1975. A schema theory of discrete motor skill learning. Psychological review 82, 4 (1975), 225.

25. John B Shea and Robyn L Morgan. 1979. Contextual interference effects on the acquisition, retention, and transfer of a motor skill. Journal of Experimental Psychology: Human Learning and Memory 5, 2 (1979), 179. 
26. Lior Shmuelof, John W Krakauer, and Pietro Mazzoni. 2012. How is a motor skill learned? Change and invariance at the levels of task success and trajectory control. Journal of neurophysiology 108, 2 (2012), 578-594. DOI : http://dx . doi .org/10.1152/jn. 00856. 2011

27. Noé Soulier. 2016. Actions, mouvements et gestes. Centre national de la danse.

28. Viren Swami and Amy Sunshine Harris. 2012. Dancing Toward Positive Body Image? Examining Body-Related Constructs with Ballet and Contemporary Dancers at Different Levels. American Journal of Dance Therapy 34, 1 (01 Jun 2012), 39-52. DOI :

http://dx.doi .org/10.1007/s10465-012-9129-7
29. Pierre Vermersch. 1996. L'explicitation de l'action. Cahiers de linguistique sociale 3 (1996), 113-120.

30. V Wilmerding and D Krasnow. 2009. Motor learning and teaching dance. Resource paper for International Association for Dance Science and Medicine (2009).

31. Daniel M Wolpert and J Randall Flanagan. 2016. Computations underlying sensorimotor learning. Current opinion in neurobiology 37 (2016), 7-11. DOI : http: //dx . doi . org/10.1016/j . conb. 2015.12.003

32. Craig Wrisberg and Zhan Liu. 1992. The Effect of Contextual Variety on the Practice, Retention, and Transfer of an Applied Motor Skill. 62 (01 1992), 406-12. 